\title{
An update on the African polio outbreak
}

oliovirus invades the nervous system, resulting in paralysis and occasionally death. In 1988, a global initiative was launched by the WHO and partners to eradicate the virus by mass immunization. The effort was largely successful; the number of polio cases fell by $99 \%$, and today the virus remains endemic to just four countries: India, Pakistan, Afghanistan, and Nigeria (1). However, in November and December of last year, hundreds of polio victims were identified in the Congo, and an estimated $40 \%$ of those cases were fatal. The JCI recently spoke to Vincent Racaniello, selfdescribed "virus guy" and polio expert at Columbia University, about the outbreak.

JCI: Is the poliovirus currently circulating in Africa different from that seen in the rest of the world?

Racaniello: There is no evidence that the poliovirus circulating in Africa (specifically in the Congo) has changed in any way. The Congo strain appears to have come from India via Angola, possibly spread by migrant workers. It is a wild-type 1 strain, not a vaccine-derived strain that has caused outbreaks in other countries.

$J C I$ : Most of the victims in the Congo are teenagers and young adults, and the fatality rate is high; does that signal that the virus has changed in some way?

Racaniello: The high prevalence of infection among teenagers and young adults is likely related to gaps in immunization. The last case of polio in the Congo was in 2000; hence immunization has lagged. When polio is acquired at a later age, the disease tends to be more severe.

JCI: How far do you think this latest outbreak is likely to spread?

Racaniello: Mass immunizations will probably restrict the outbreak to the Congo. However, consider that only $1 \%$ of poliovirus infections are paralytic. That means in the current outbreak, where 476 cases of paralytic disease have been record$\mathrm{ed}$, there have been a total of 47,600 infections, $99 \%$ of which have gone unnoticed. Therefore the potential for spreading the infection to neighboring countries is high - unless those countries maintain high levels of immunization. Migrant worker travel can also cause distant spread of infection. In 2004, there was an outbreak of polio in Nigeria; the virus spread to other countries in Africa and then to Indonesia.
JCI: How should vaccination policy change to prevent future outbreaks?

Racaniello: All countries need to maintain high levels of immunization coverage until the disease has been eradicated. In the US and other developed countries, this is done by routine immunization at birth. In underdeveloped countries, this is difficult due to poor health care systems, unstable political situations, and armed conflict. Hence, mass immunizations are used to improve coverage. As long as countries such as the Congo allow immunizations to lag, there will be outbreaks.

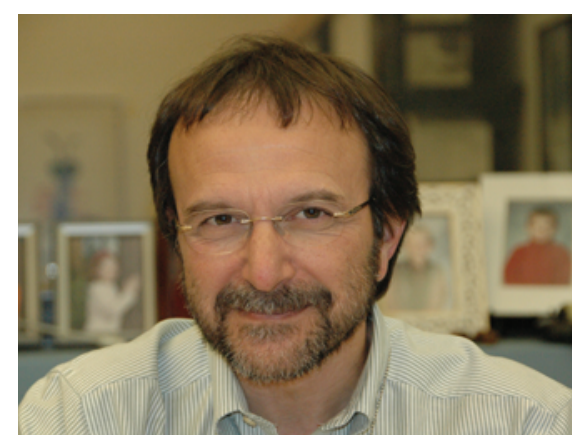

Vincent Racaniello

JCI: What treatment options exist for infected patients, and how available are those therapies in the affected countries?

Racaniello: When polio is fatal, it is mainly because of respiratory paralysis. Mechanical ventilation is required to support the patient - which is typically not widely available in underdeveloped countries. The problem is compounded in the Congo because of armed conflict.

JCI: What can we learn about the virus itself from this most recent outbreak?

Racaniello: As long as there is circulating poliovirus somewhere in the world, it will find unimmunized individuals. Sequencing of the isolates should reveal whether any changes have occurred that might be associated with increased virulence and also should allow tracking of the origin of the virus.

JCI: How could further research on poliovirus pathogenesis change approaches to polio prevention or treatment?

Racaniello: We could use extremely rapid diagnosis. The problem now is that when paralysis is detected, it is too late to try to treat the infection. If we could eas- ily screen entire populations for the virus, it could give earlier clues, which might then allow treatment. But we don't have any antivirals that would be useful. No one is willing to develop such compounds because there is no rapid diagnostic! Plus, it doesn't make commercial sense to develop such compounds if polio is going to be eradicated. Clearly the wealthier governments need to support such work as an altruistic gesture for the rest of the world. There are many new approaches to therapy - RNAi, for example - that otherwise just won't be explored.

JCI: How has the study of poliovirus been instructive in our understanding of virology more generally?

Racaniello: Polio has been huge for virology in general; poliovirus has established paradigms for the study of viruses since the 1940s. It was among the first RNA viruses to be studied. It replicates easily and so allowed scientists to study viral replication in cultured cells. In the late 1980s, a transgenic mouse model for polio was developed - the first for any virus - allowing studies of the pathogenesis of disease in a convenient animal model. But now polio research is dying because of the perception that it will soon be eradicated. In fact, this perception has influenced study sections in the US for the past 10 years - yet we have not seen eradication. It's safe to say that polio research has been killed by the talk of eradication long before such eradication was even close.

JCI: How far do you think we are from global polio eradication?

Racaniello: How long it will take to eradicate is impossible to predict because the eradication curve is asymptotic. India has been a problem for many years, but in 2010 the number of cases are down dramatically. In 2009, there were 741 paralytic cases in India, and so far in 2010 there have been 40. If that trend continues, it will be a good sign. Eradication means no global cases for at least 5 years, certainly no less than 10 years. I can't imagine that the level of immunization can be sustained at the required levels for that long in all countries.

\section{Kathryn Claiborn}

1. WHO poliomyelitis fact sheet. World Health Organization Web site. http://www.who.int/mediacentre/ factsheets/fs114/en/index.html. Accessed December 20, 2010. 